Continued from previous page

is well defined, because it is not necessary to duplicate an entire computer system many times in order to achieve parallelism.

We have designed and constructed an experimental parallel processor at the State University of New York at Stony Brook which can achieve a large degree of parallelism using a modular format for the arithmetic processors and their associated memories. Necessary communication between the several processors is made over multiple, very high-speed computer links which run at full speed simultaneously. The couple parallel computing elements are run under synchronous control of a single host computer which sends the compu- tational routines to the processors before a computing session begins and which also provides data for the computation, such as particle forces and initial positions. As each task is completed, such as updating the positions and velocities of about 20 particles for each processor, the host control system redirects each processor to a new task or a new group of particles. This process takes about one microsecond. In most cases, all processors are redirected within the same microsecond.

Our test results show that computational capabilities can be achieved in a relatively inexpensive machine which greatly exceed the speed of super-minicomputers which cost a hundred times as much. For example, a 16-processor parallel computing engine with a modest host computer will cost about $\$ 55,000$ and has the computational speed of 80 million instructions per second. In addition, the modular nature of the design makes it possible to connect as many 16-processor modules together as the problem warrants with only a slight change in the software. When these features are combined with the full-time availability of this computer to the scientist because of its low cost it can be seen that a completely new range of simulation becomes practically possible.

\title{
Leamy Receives 1985 Woody Award
}

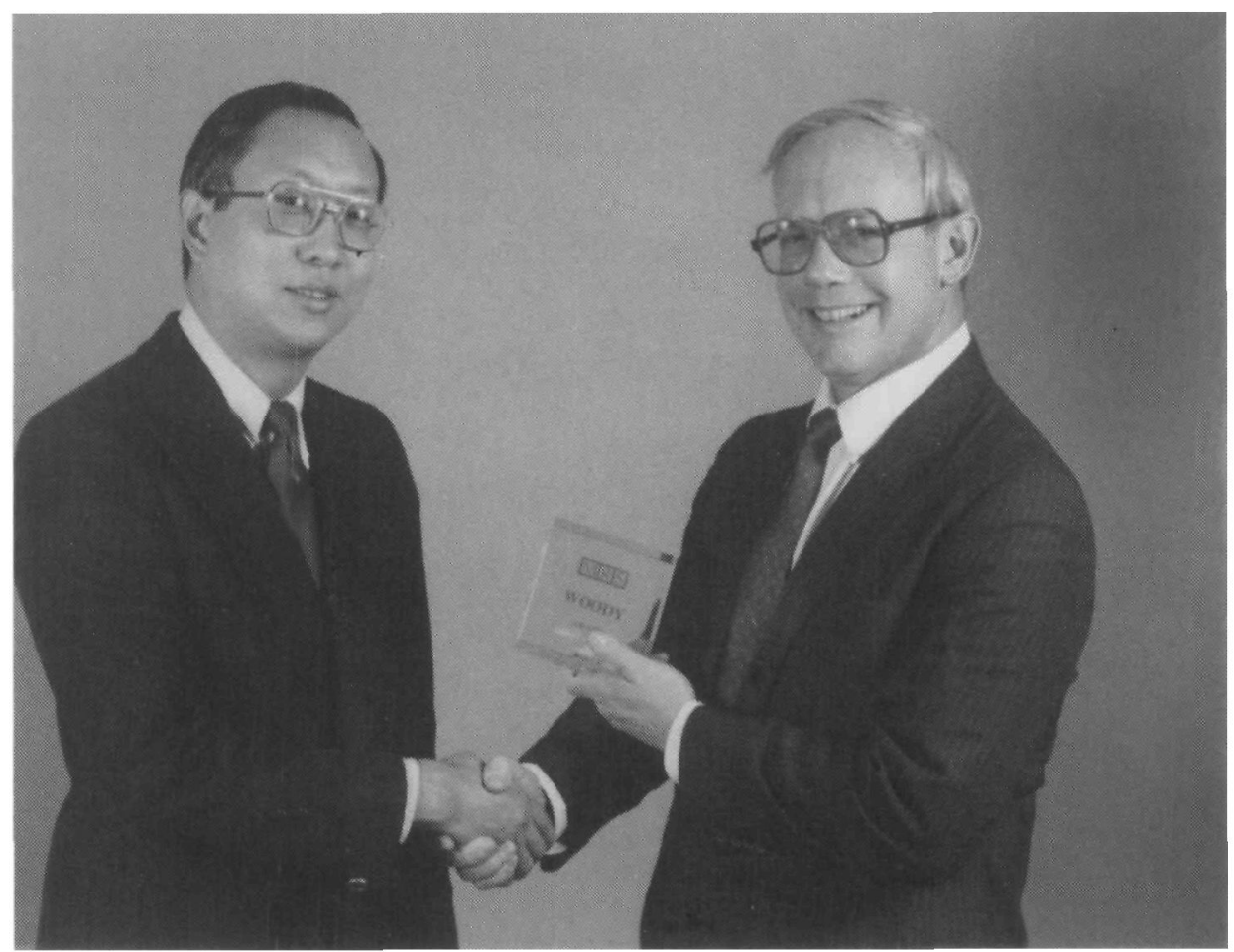

Harry Leamy (right) receives 1985 Woody Award from Bob Chang.

Awards Committee Chair Woody White announced to the Council during the Fall Meeting in Boston that Harry J. Leamy, AT\&T Bell Laboratories, was the 1985 recipient of the MRS Woody Award. The award is bestowed annually to a member of the Society for outstanding organizational talents, energy, and service to the Society.

R. P. H. Chang presented the award to Leamy in early January at Bell Labs. Speaking on behalf of the Society, Chang said, "The Council, Otticers and membership have benefited a thousand-fold from your contributions. The award is but a small token of their appreciation for the critical role you played in starting the MRS BULLETIN and the conference proceedings series, launching the effort which resulted in the MRS Headquarters, starting the Laser Annealing Symposium, serving as meeting chairman for the highly successful 1981 Boston Meeting, and all the many contributions made as President in 1983, Vice President in 1982, and Chairman of the Publications, Long Range Planning, Awards, and Nominating Committees at various times during the last five years."

"Above all," Chang said, "the award is given for your incredible ability to attract other dedicated individuals to become actively involved in the Materials Research Society."

Ironically, it was Leamy who instituted the Woody Award over a year ago to recognize individuals whose dedication to materials research is exemplified by outstanding service to the Society. The award is named after its first recipient Woody White, 1984 MRS President. 